\title{
High Throughput Quantitative Bioanalytical LC/MS/MS Determination of Gemifloxacin in Human Urine
}

\author{
Adnan A. Kadi, ${ }^{1}$ Rihab F. Angawi, ${ }^{2}$ Mohamed W. Attwa, ${ }^{1}$ \\ Hany W. Darwish, ${ }^{1,3}$ and Ali Saber Abdelhameed ${ }^{1}$ \\ ${ }^{1}$ Department of Pharmaceutical Chemistry, College of Pharmacy, King Saud University, P.O. Box 2457, Riyadh 11451, Saudi Arabia \\ ${ }^{2}$ Department of Chemistry, College of Science, King Abdulaziz University, P.O. Box 54881, Jeddah 21589, Saudi Arabia \\ ${ }^{3}$ Department of Analytical Chemistry, Faculty of Pharmacy, Cairo University, Kasr El-Aini Street, 11562 Cairo, Egypt
}

Correspondence should be addressed to Ali Saber Abdelhameed; asaber@ksu.edu.sa

Received 13 September 2013; Accepted 8 November 2013

Academic Editor: Alberto Ritieni

Copyright (C) 2013 Adnan A. Kadi et al. This is an open access article distributed under the Creative Commons Attribution License, which permits unrestricted use, distribution, and reproduction in any medium, provided the original work is properly cited.

\begin{abstract}
A highly specific, sensitive, and rapid method, to quantify gemifloxacin in human urine using HPLC coupled to the triple quadrupole mass spectrometer system, was developed and validated. Gemifloxacin and ofloxacin (internal standard) were rapidly extracted from urine samples without any tedious pretreatment procedure. Urine samples were filtered through a Millex-GP, $0.22 \mu \mathrm{m}$ syringe filter. Optimal chromatographic separation of the analytes was achieved on Zorbax SB-C $\mathrm{C}_{18}(30 \mathrm{~mm} \times 2 \mathrm{~mm}$ i.d., $3.5 \mu \mathrm{m}$ maintained at ambient temperature). The mobile phase consisted of $0.1 \%$ formic acid (pH 3.2) and acetonitrile ( $80: 20)$ and a flow rate of $0.2 \mathrm{~mL} \mathrm{~min}^{-1}$ for $4 \mathrm{~min}$. The analytes were monitored by electrospray ionization in positive ion multiple reaction monitoring mode. The method provided a linear response $(r=0.9998)$ from a quantitation range of $5 \mathrm{ng} \mathrm{mL}^{-1}$ to at least $500 \mathrm{ng} \mathrm{mL} \mathrm{m}^{-1}$. The mean extraction recovery $\%$ of gemifloxacin from spiked human urine was $101.33 \pm 2.58 \%$. The reproducibility of the method was reliable with the intra- and inter-day precision of $<2 \%$ and accuracy within $2 \%$. The established method was reliably applied for the determination of gemifloxacin in volunteers' urine samples with the mean recoveries of gemifloxacin from Factive tablets $320 \mathrm{mg}>97.0 \%$.
\end{abstract}

\section{Introduction}

Fluoroquinolones are one of the most promising and intensively studied drugs of contemporary anti-infective chemotherapy. Since the introduction of nalidixic acid, the first quinolone, in 1962 [1], structural modifications have resulted in the production of numerous agents in second-, third-, and fourth-generation fluoroquinolones with improved antibacterial activity and pharmacological properties. In April 2003, the US Food and Drug Administration (FDA) approved gemifloxacin (GFX) as a fourth generation fluoroquinolone. Gemifloxacin, (R,S)-7-[(4Z)-3(aminomethyl)-4-(methoxyimino)-1-pyrrolidinyl]-1-cyclopropyl-6-fluoro-1,4-dihydro-4-oxo-1,8-naphthyridine-3-carboxylic acid (SB-265805; LB-20304a) (CAS no. 175463-14-6) (Figure 1), is used as an antibacterial agent against both gram-positive bacteria and gram-negative bacteria [2] for the treatment of community-acquired respiratory tract infections such as pneumonia and acute bacterial exacerbations of chronic bronchitis [3-5]. Although it is not yet official in any of the pharmacopeia, GFX has shown potent activity against major pathogens involved in respiratory as well as urinary tract infections. Those pathogens include Streptococcus pneumonia, Haemophilus influenzae, Moraxella catarrhalis [6] and the atypical organisms; Legionella pneumophila, chlamydia species, mycoplasma species, Escherichia coli, Klebsiella and Staphylococcus saprophyticus [7-12]. GFX has a dual activity; it inhibits both DNA gyrase and topoisomerase IV which are responsible for maintaining the integrity of the supercoiled DNA double helix during replication and transcription events. Hence, the interference with their normal action culminates in rapid bacterial death, as well as limited emergence of fluoroquinolone resistance $[12,13]$.

A detailed understanding of correlations of drug levels with drug action is an important aspect of the routine use of drugs. The accurate quantification of agents in biological 


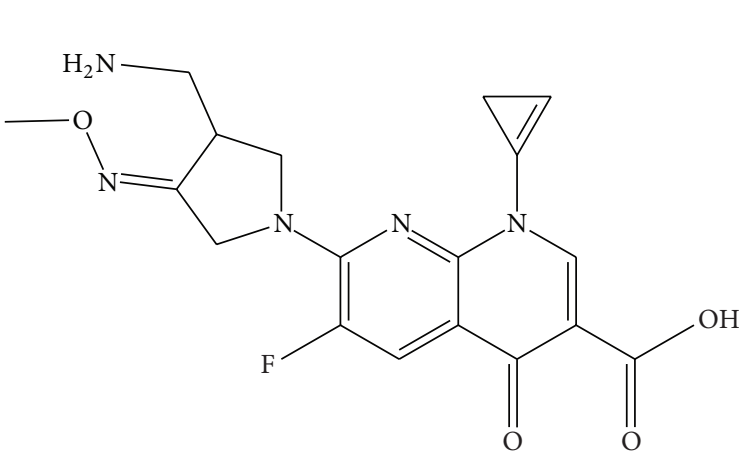

(a)

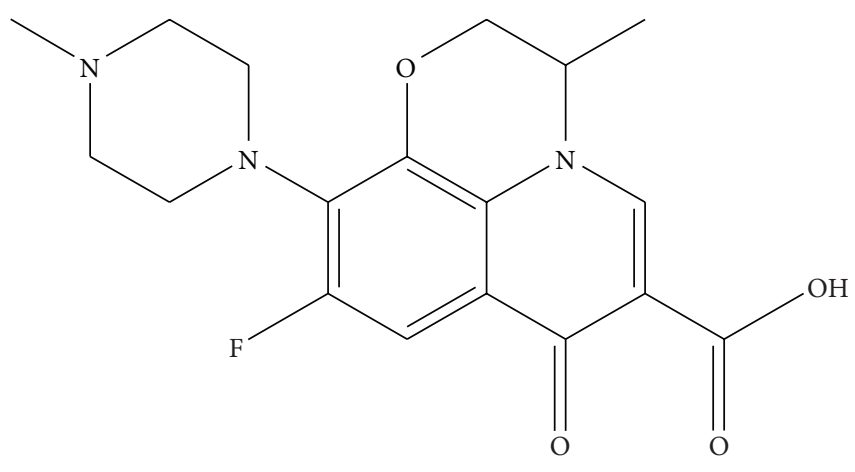

(b)

Figure 1: Chemical structure of (a) gemifloxacin (GFX) and (b) ofloxacin (OFX; IS).

matrices such as blood, serum, urine, and tissue samples is the cornerstone of therapeutic drug monitoring. Clinical decisions and further treatment options for patients can only be based on accurate validated analytical methods. Likewise, when determining the efficacy, dose limiting toxicity or pharmacokinetics of new agents and/or new combinations of agents and accurate and precise quantification methods are required. Therefore, detailed specific, reproducible and accurate method for the quantitation of GFX is necessary. Additionally, examining the matrix effects represents an important issue in LC-MS/MS, particularly when dealing with biological matrices such as urine. These phenomena can be reduced by efficient sample preparation [14] and an adequate chromatographic separation with the elution of the analytes outside the matrix effect time window generally observed at the beginning of the chromatogram [15]. However in quantitative analysis these conditions might be insufficient to reduce interference, which calls for the integration of other approaches such as multiple reaction monitoring (MRM) mode to compensate for residual matrix effects.

An extensive literature survey revealed that several analytical methods have been reported for the determination of gemifloxacin in pharmaceutical preparations or human plasma or even in honey. Such methods are the various spectrophotometric techniques [16-25], capillary electrophoresis [26], high performance liquid chromatography [27-36], LC-mass spectrometry [37-41], microchip electrophoresis [42], chemiluminescence [43] and potentiometry [44] These methods were related to some major drawbacks such as having inadequate sensitivity, being time-consuming, tedious and sophisticated sample preparation. However, to the best of our knowledge, determination of GFX with complete validation in human urine has never been published.

The present study describes a novel method for the quantitative determination of GFX in human urine. It is simple, rapid, specific and highly sensitive that employs an isocratic LC-MS/MS with high sample throughput. The method is fully validated with sufficiently precise results. The proposed method was aimed at developing an easy and rapid eco-friendly assay method for GFX without any timeconsuming sample preparation steps for routine analysis.
Moreover, this method overcomes the need to purchase expensive internal standard such as isotopic labeled GFX [38]. In the proposed LC-MS/MS method, minimal sample clean-up without tedious extraction procedure is achieved as there is no need to extract GFX from the urine, thereby minimizing the error in quantitation. The urine samples can be directly used after filtration through syringe filters and then adequate dilution of supernatant liquid.

\section{Materials and Methods}

2.1. Chemicals and Reagents. Gemifloxacin mesylate and Ofloxacin reference standards (claimed purity, $>98 \%$ for both) were purchased from Sigma-Aldrich (St. Louis, MO, USA). Bidistilled water was purified using cartridge system (Milford, Bedford, USA) (ultra-pure water of $18 \mu \Omega$ was obtained from Milli-Q plus purification system, Millipore, Waters (Millipore, Bedford, MA, USA). HPLC-grade solvents and reagent-grade formic acid were purchased from $(\mathrm{BDH}$, England) and all other chemicals and reagents were of analytical grade (Riedel de Haën, Germany).

2.2. Instrumentation and Conditions. Chromatographic separation was performed on an Agilent 1200 series system consisting of G1311A binary pump, G1322A degasser, G1367B HIP-ALS autosampler, G1316 thermostated column compartment and G1315 DAD detector and an Agilent 6410 QqQ LC/MS (Agilent Technologies, Palo Alto, CA, USA). Binary chromatography was carried out on Agilent eclipse plus $\mathrm{C}_{18}$ analytical column $(30 \mathrm{~mm} \times 2 \mathrm{~mm}, 3.5 \mu \mathrm{m})$ (Agilent Technologies, Palo Alto, CA, USA) column temperature was kept constant at $25 \pm 2^{\circ} \mathrm{C}$. The most suitable chromatographic conditions were achieved at a flow rate of $0.2 \mathrm{~mL} \mathrm{~min}^{-1}$ with a mobile phase consisted of $80 \% 0.1 \%$ Formic acid, and an apparent $\mathrm{pH}$ adjusted to 3.2 with the addition of ammonium formate: and $20 \%$ acetonitrile. Sample injection volume was $10 \mu \mathrm{L}$ with a total run time of 4 minutes. Detection was performed on a QqQ MS detector, operated with an ESI interface in the positive ionization mode. Nitrogen was used as desolvation gas at a flow rate of $11 \mathrm{~L} / \mathrm{min}$ and high purity nitrogen as collision gas at a pressure of 50 psi. Source temperature was set at $350^{\circ} \mathrm{C}$ and capillary 
voltage at $4 \mathrm{kV}$, dwell time for each ion was $200 \mathrm{~ms}$. Quantification was achieved using multiple reaction monitoring $(\mathrm{MRM})$ of the transitions $390.1 \rightarrow 372.1$ and $390.1 \rightarrow 313$ for GFX and $362.1 \rightarrow 318$ and $362.1 \rightarrow 261$ for OFX (IS). These transitions were previously reported in other publications for the detection of the studied compounds [37, 38, 40]. Fragmentor voltage was set to 140 and $160 \mathrm{~V}$ with collision energy of 15, 20 and 15, $25 \mathrm{~V}$ for GFX and OFX, respectively. MassHunter software (Agilent Technologies, Palo Alto, CA, USA) was used to control the instruments and data acquisition.

2.2.1. Preparation of Standard Solutions. Standard solutions preparation was conducted at room temperature under subdued light under sodium lamp. GFX standard solution was prepared in $50 \%$ ethanol in deionized (DI) water to produce a final concentration of $1.0 \mathrm{mg} \mathrm{mL}^{-1}$. The working standard solution was prepared by diluting $1.0 \mathrm{~mL}$ of stock solution to $10 \mathrm{~mL}$ with $50 \%$ ethanol in DI water to give a $100 \mu \mathrm{g} \mathrm{mL}^{-1}$ concentration. One $\mathrm{mL}$ of the working standard solution was diluted with $9 \mathrm{~mL}$ of mobile phase to produce working standards of $10 \mu \mathrm{g} \mathrm{mL}^{-1}$. Stock standard solution of the internal standard, OFX, was prepared in $0.05 \mathrm{M}$ acetic acid in DI water to produce a concentration of $100 \mu \mathrm{g} \mathrm{mL}^{-1}$. $200 \mu \mathrm{L}$ of this stock solution was diluted to $10 \mathrm{~mL}$ with mobile phase producing a working solution of $2 \mu \mathrm{g} \mathrm{mL}^{-1}$.

Working solution was protected from light with aluminum foil wrapping and stored at $-70^{\circ} \mathrm{C}$ until being required for analysis.

2.2.2. Sample Preparation Procedure and Construction of the Calibration Curve. Appropriate volumes of gemifloxacin (GFX) working standard solution $\left(10 \mu \mathrm{g} \mathrm{mL}^{-1}\right)$ were added to drug-free human urine $(20 \mathrm{~mL})$ to prepare eight nonzero concentrations: 50,150, 250,500,1500, 2000, 4000 and $5000 \mathrm{ng} \mathrm{mL}^{-1}$. One portion of each concentration was diluted with 9 portions of the mobile phase to produce 5, 15 (low quality control: LQC), 25, 50, 150 (medium quality control: MQC), 200, 400 (high quality control: HQC) and $500 \mathrm{ng} \mathrm{mL}^{-1}$, respectively. A volume of $3 \mathrm{~mL}$ of each sample was filtered through a Millex-GP, $0.22 \mu \mathrm{m}$ syringe filter (Millipore, Billerica, MA, USA), the filtered diluted urine samples were then loaded in the autosampler tray and volumes of $10 \mu \mathrm{L}$ were injected into the chromatographic system. The drug-free urine was processed with similar procedure using deionized water instead. Blank urine was then tested to ascertain the absence of any endogenous interference at the retention time of GFX and OFX. An eight-point calibration curve $\left(5,15,25,50,150,200,400\right.$ and $\left.500 \mathrm{ng} \mathrm{mL}^{-1}\right)$ was constructed by plotting the peak area ratio of GFX to OFX (IS) ( $y$-axis) versus GFX nominal concentrations ( $x$-axis). Analysis of calibration samples at each concentration was performed in triplicates. Slope, intercept, and $r$ values were calculated as regression parameters by linear regression. The linear regression equation was used to calculate the concentrations of GFX in spiked urine based on their peak area ratios.
2.3. Method Validation. The proposed analytical method was fully validated in terms of sensitivity, linearity, stability, selectivity, accuracy, intraday and interday precision and system suitability. The method validation was developed based on the criteria established by the recommendations of the International Conference on Harmonisation (ICH) [45].

2.3.1. Specificity. Specificity is the ability of a method to determine accurately the analyte of interest in the presence of other components in a sample matrix under the stated conditions of the test [46]. To demonstrate the specificity of the analytical procedure, ten different blank urine samples obtained from various healthy volunteers were analyzed for the peaks interfering with the detection of the analytes or internal standard. Possible carryover effects were reduced via the use of MRM mode and increasing run time after elution of the analytes.

2.3.2. Linearity and Sensitivity. Using the aforementioned optimum chromatographic conditions, three independent calibration curves were constructed correlating the calculated peak area ratio of GFX to the internal standard (OFX) versus the nominal concentrations of GFX. Calibration plots for GFX in urine samples were prepared daily at eight concentration points; each concentration was injected in triplicate. Regression analysis for the results was carried out using the least-square method (see Section 2.2.2).

2.3.3. Limit of Detection and Lower Limit of Quantitation. The lower limit of quantitation (LOQ) is the lowest concentration of the standard curve which can be measured with acceptable accuracy and precision for the analyte from the normal human urine. The limit of detection (LOD) and the lower limit of quantitation (LOQ) were calculated based on the following equations:

$$
\mathrm{LOD}=3.3 \frac{\sigma}{\mathrm{S}}, \quad \mathrm{LOQ}=10 \frac{\sigma}{\mathrm{S}},
$$

where $\sigma$ is the standard deviation of the intercept of regression line and $S$ is the slope of regression line of the calibration curve [45].

2.3.4. Precision and Accuracy. Intraday accuracy and precision evaluations were performed by repeated analysis of GFX in human urine. The run consisted of a calibration curve plus six replicates of each low, medium and high quality control (QC) samples $\left(15,150,400 \mathrm{ng} \mathrm{mL}^{-1}\right)$. Interday accuracy and precision were assessed by analysis of samples consisting of a calibration curve and six replicates of low, medium, and high quality control samples for GFX on three successive days. The overall precision of the method expressed as relative standard deviation and accuracy of the method expressed in terms of $\%$ error $=[$ (mean measured concentration nominal concentration)/nominal concentration] $\times 100$.

2.3.5. Robustness and Ruggedness. In order to measure the extent of the method robustness, the most critical parameters were interchanged while keeping the other parameters 
unchanged, and in parallel, the chromatographic profile was observed and recorded. The chromatographic parameters varied around the optimum value in the method to reflect changes likely to arise in different test environments. The studied parameters were the composition of the mobile phase, $\mathrm{pH}$, and flow rate. Ruggedness of the method was determined with the aid of two different analysts and using mobile phase components from two different manufacturers.

2.4. Application of the Method. The proposed analytical method was used for the determination of GFX in human urine samples. Twenty six volunteers (mean age, $31.7 \pm$ 2.8 years; mean weight $70.2 \pm 6.3$ kilograms) provided informed consent. Factive tablet equivalent to $320 \mathrm{mg}$ gemifloxacin/tablet (Oscient Pharmaceuticals, USA) was administered to each volunteer. Urine samples $(5 \mathrm{~mL})$ were collected from each volunteer prior to the administration and 2 , $4,6,8,12$ and $24 \mathrm{~h}$ after administration. Urine samples were then filtered through Millex-GP, $0.22 \mu \mathrm{m}$ syringe filter (Millipore, Billerica, MA, USA) and analyzed directly without pretreatment or filtered into a collection tube and stored at $-70^{\circ} \mathrm{C}$ until analysis. The study protocol was approved by the ethics committee of the College of Pharmacy and the Institutional Review Board (IRB) of King Khalid University Hospital, King Saud University, Saudi Arabia.

\section{Results and Discussion}

3.1. Chromatographic Separation and Mass Spectrometry. The chromatographic conditions, especially the composition of the mobile phase, were optimized through several trials to achieve optimum resolution, high sensitivity, and symmetrical peak shape for GFX and IS. Different percentages of acetonitrile and formic acid were tested. The addition of formic acid to the mobile phase facilitated the ionization of the analytes, enhanced ion response, and modified peak shape. A mixture of $0.1 \%$ formic acid-acetonitrile $(80: 20$, $\mathrm{v} / \mathrm{v} ; \mathrm{pH} 3.2$ ) was used isocratically at retention times of 2.05 and 0.98 minutes for GFX and IS, respectively, using the optimized LC-MS/MS condition and with no interference by endogenous compounds. The elution of the GFX and internal standard was achieved within 4 minutes. Under the aforementioned assay conditions, the examined drug was well resolved, and carry over was not obvious in either blank matrices or zero-level standard (blank with IS). A typical chromatogram of standard solutions is presented in Figure 2. Precision of retention times was examined to evaluate the system suitability. Intraday repeatability (mean value of five measurements at three concentration levels, $n=15$ ) and inter-day precision (mean value of three measurements at three concentration levels during five days, $n=$ 45) of GFX retention time revealed RSD values less than $1 \%$.

Additionally, mass spectrometric conditions were optimized to achieve the maximum stable response, of the parent ions and the major product ions of the analytes. Multiple reaction monitoring (MRM) afforded by MS/MS had a greater advantage in reducing interference and enhancing

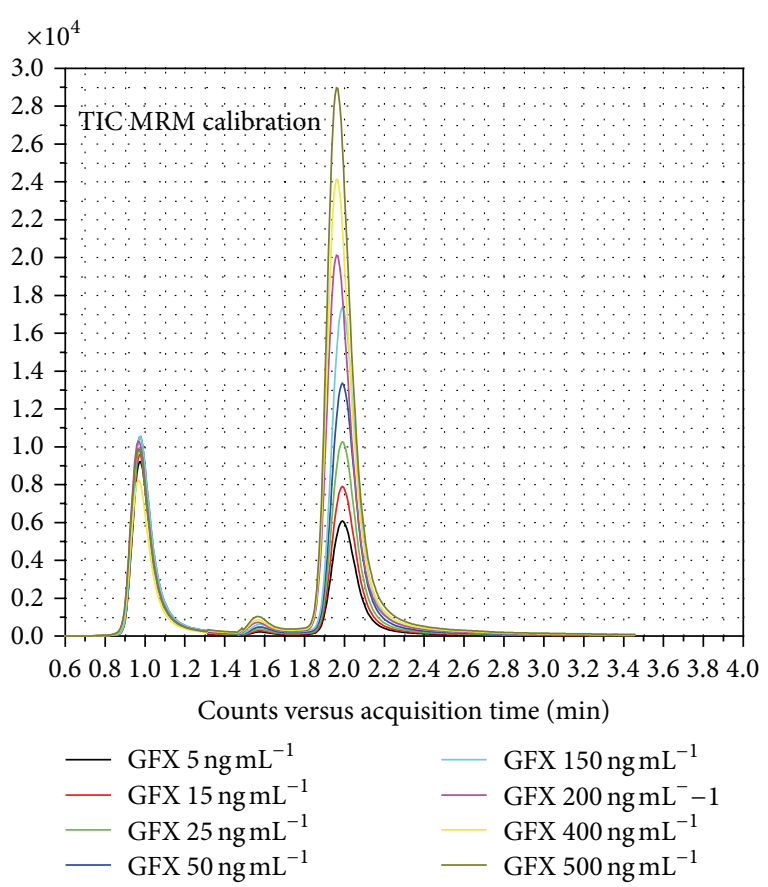

FIGURE 2: Total ion chromatogram of MRM scan of GFX and IS in spiked urine.

sensitivity over selected ion monitoring (SIM). ESI was operated in positive ion mode for the LC-MS/MS analysis to provide optimum sensitivity and selectivity. The positive ion spectrum for GFX and OFX consisted predominately of a single ion at $\mathrm{m} / \mathrm{z} 390.1$ and 362.1, respectively, which correspond to the protonated molecular (parent) ion. By isolating $\mathrm{m} / \mathrm{z} 390.1$ (GFX) and 362.1(OFX), in the first mass analyzer (Q1), and inducing fragmentation (q2), the major ion observed in the product spectrum was detected at $\mathrm{m} / \mathrm{z}$ 313 and 372.1 for GFX and at $\mathrm{m} / z 318$ and 261 (Figure 3). The major ion for GFX $(\mathrm{m} / \mathrm{z} 313)$ produced significant signal intensity relative to any other ion's observed in the spectrum and was therefore selected for sensitive quantification of GFX. Another characteristic ion $(\mathrm{m} / \mathrm{z}$ 372.1) was used as a qualifier ion in quantitative software of Agilent $6410 \mathrm{QqQ}$. The major ion observed in the product spectrum of OFX was detected at $\mathrm{m} / \mathrm{z} 318$ and was chosen for sensitive quantification of OFX. Another characteristic ion $(\mathrm{m} / \mathrm{z} 261)$ was used as a qualifier ion in quantitative software of Agilent $6410 \mathrm{QqQ}$.

3.2. Method Validation. The analytical procedure is simple and the method has high sample throughput. This uncomplicated approach is possible because of the selectivity of tandem mass spectrometry which allows accurate measurement at levels as low as $5 \mathrm{ng} \mathrm{mL}^{-1}$ with minimal sample cleanup avoiding any tedious extraction procedure. This method showed fast separation time (4 minutes) compared to previous reported methods (12.5 or 5 minutes) $[37,38]$ as well as a very low flow rate $\left(0.2 \mathrm{~mL} \mathrm{~min}^{-1}\right)$ compared to $0.6 \mathrm{~mL} / \mathrm{min}$ or $1 \mathrm{~mL} \mathrm{~min}^{-1}[37,38]$. This yielded more eco-friendly elution with reduced consumption of organic solvent in addition 


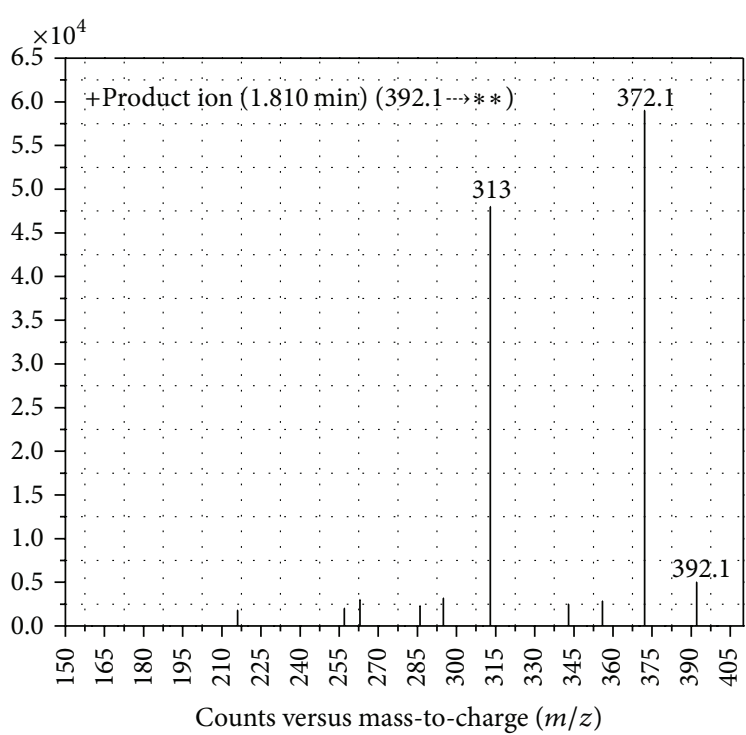

(a)

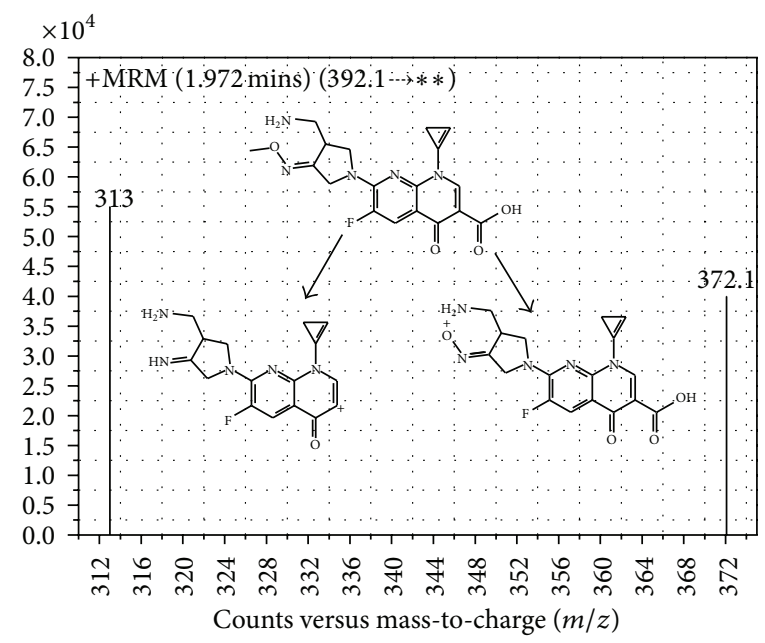

(c)

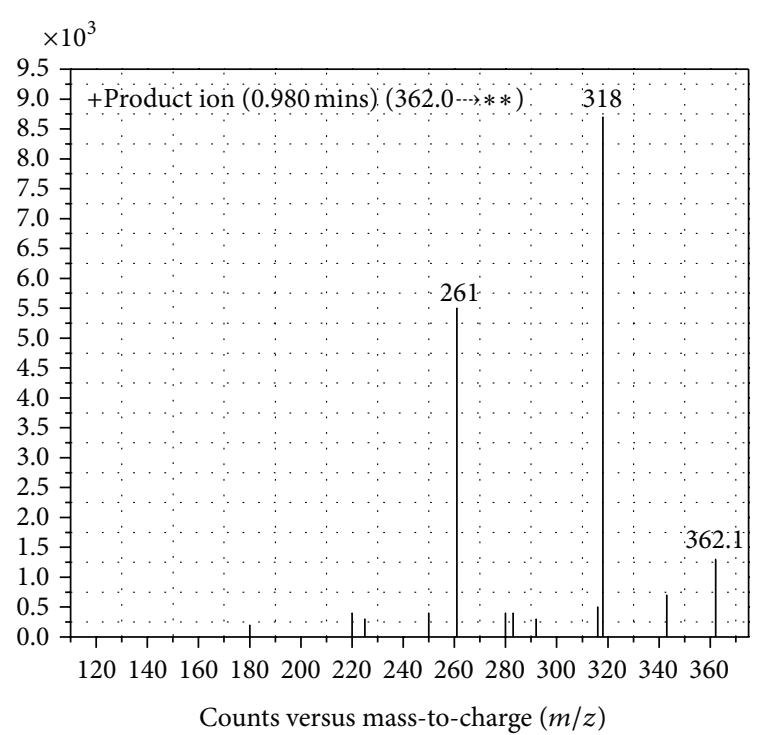

(b)

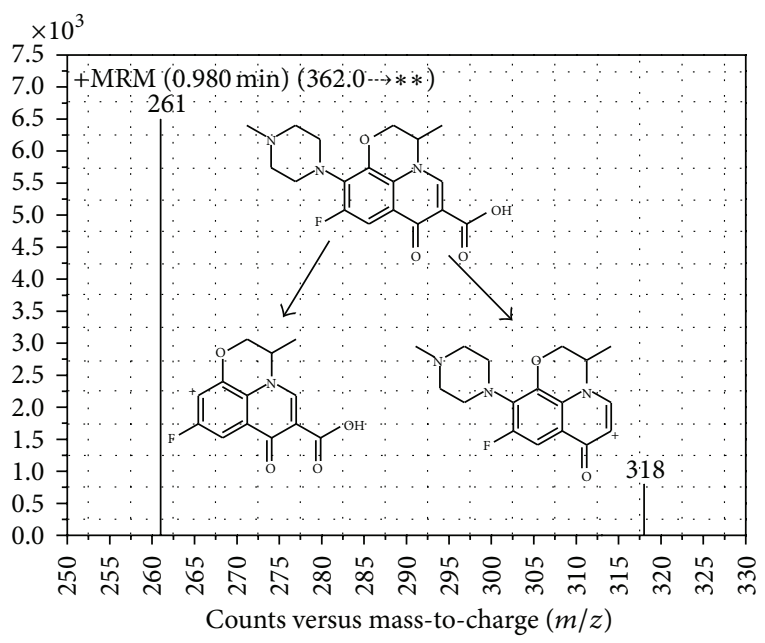

(d)

FIGURE 3: MS/MS spectrum of (a) GFX (b) OFX (IS), MRM Spectra of (c) GFX and (d) OFX (IS).

to cost reduction for running the method. The method is applied to the urine of healthy volunteers to monitor urinary excretion of GFX.

3.2.1. Specificity. The method is specific as no interference was observed in drug-free urine samples at the retention time of GFX. Additionally, no carry-over effect was observed in the system. GFX and IS were well separated under the HPLC conditions applied and retention times were 2.05 and $0.98 \mathrm{~min}$, respectively.

3.2.2. Linearity and Sensitivity. The method is validated according to the $\mathrm{ICH}$ guidelines [45]. It is rugged and adequately sensitive for routine subject sample analysis. The linear regression analysis for the results was carried out using the least-square method. The relative standard deviation values of each concentration point (triplicates) did not exceed
$5.09 \%$. The results revealed a good linear calibration fit in the range of $5-500 \mathrm{ng} \mathrm{mL}^{-1}$, with a correlation coefficient $(r) \geq 0.999$. A typical calibration curve has the regression equation of $y=1.2636 x+5.2058\left(r^{2}=0.9998\right)$. The high $r^{2}$ value was indicative of the good linearity, and the low values of standard deviations of the intercept and the slope were indicative of the significant validity of the calibration points used for constructing the calibration curve.

\subsubsection{Limit of Detection and Lower Limit of Quantification.} The lower limit of quantification (LOQ) was $4.27 \mathrm{ng} \mathrm{mL}^{-1}$. The lower limit of detection (LOD) was $1.28 \mathrm{ng} \mathrm{mL}^{-1}$. Table 1 summarizes the back-calculation of GFX concentration of the calibration standards in human urine. The accuracy and precision for the analyte covering the concentration range of $5-500 \mathrm{ng} \mathrm{mL}^{-1}$ ranged from 98.09 to 104.69 and 0.46 to $3.52 \%$, respectively. 
TABLE 1: Data of back-calculated GFX concentration of the calibration standards in human urine.

\begin{tabular}{lcccc}
\hline Nominal concentration $\left(\mathrm{ng} \mathrm{mL}^{-1}\right)$ & Mean $^{\mathrm{a}}$ & Standard deviation $(\mathrm{SD})$ & Precision $(\%)$ & Accuracy $(\%)$ \\
\hline 5 & 4.97 & 0.12 & 2.43 & 1.59 \\
15 & 14.78 & 0.24 & 2.43 & 98.43 \\
25 & 25.53 & 0.62 & 3.52 & 102.11 \\
50 & 52.35 & 1.84 & 2.03 & 104.69 \\
150 & 156.75 & 3.18 & 2.11 & 104.50 \\
200 & 205.59 & 4.34 & 0.69 & 102.79 \\
400 & 392.39 & 2.73 & 0.46 & 98.09 \\
500 & 502.41 & 2.33 & & 100.48 \\
\hline
\end{tabular}

${ }^{a}$ Average of six determinations.

TABLE 2: Intraday and interday precision and accuracy of GFX (QC) samples.

\begin{tabular}{|c|c|c|c|}
\hline \multirow{2}{*}{ Day of analysis } & \multicolumn{3}{|c|}{ GFX measured concentration in human urine } \\
\hline & LQC (15 ng mL $\left.{ }^{-1}\right)$ & MQC $\left(150 \mathrm{ng} \mathrm{mL}^{-1}\right)$ & $\mathrm{HQC}\left(400 \mathrm{ng} \mathrm{mL}^{-1}\right)$ \\
\hline \multirow{6}{*}{ Day 1} & 14.73 & 154.57 & 400.96 \\
\hline & 14.89 & 155.05 & 404.32 \\
\hline & 15.16 & 154.58 & 399.73 \\
\hline & 14.99 & 153.24 & 402.41 \\
\hline & 14.89 & 153.60 & 402.91 \\
\hline & 14.71 & 154.78 & 403.24 \\
\hline \multirow{6}{*}{ Day 2} & 14.68 & 154.43 & 402.69 \\
\hline & 14.73 & 153.67 & 402.22 \\
\hline & 14.82 & 153.89 & 401.68 \\
\hline & 14.55 & 154.26 & 400.98 \\
\hline & 14.75 & 154.43 & 399.54 \\
\hline & 14.95 & 154.22 & 398.32 \\
\hline \multirow{6}{*}{ Day 3} & 14.89 & 153.62 & 400.25 \\
\hline & 14.56 & 154.57 & 401.15 \\
\hline & 14.77 & 154.32 & 402.66 \\
\hline & 14.63 & 153.51 & 403.56 \\
\hline & 14.48 & 153.17 & 401.12 \\
\hline & 14.85 & 153.42 & 401.23 \\
\hline Mean & 14.78 & 154.07 & 401.61 \\
\hline Standard deviation (SD) & 0.17 & 0.57 & 1.55 \\
\hline Precision $(\mathrm{CV})(\%)$ & 1.14 & 0.38 & 0.39 \\
\hline Accuracy & 98.53 & 102.72 & 100.40 \\
\hline
\end{tabular}

3.2.4. Precision and Accuracy. The precision and accuracy at the low, medium and high concentration of GFX in urine were within the acceptable limits (Table 2). Intra- and interday relative standard deviations (precision, \% CV) were in the range of 0.38 and $1.14 \%$. Accuracy was estimated as percent error (relative error) [(measured concentration spiked concentration)/spiked concentration] $\times 100$, while the precision was quantified by calculating intra- and inter-day \% CV values (Table 3). These results indicated the acceptable accuracy and precision of the method ( $\mathrm{ICH}$ Guidance for Industry, 2000).

3.2.5. Robustness and Ruggedness. The studied parameters were the composition of the mobile phase, $\mathrm{pH}$, and flow rate. The results $(\mathrm{RSD} \sim 0.2-0.4 \%$ ) indicated that small change in the conditions did not have significant effect on the determination of GFX. Additionally, robustness and ruggedness were determined by using mobile phase components from two different manufacturers and two different analysts. There was no significant change observed in the retention time of GFX; RSD was $0.26-0.38 \%$.

3.2.6. Stability. The stability of the GFX standard solutions, stored at $4^{\circ} \mathrm{C}$, was evaluated at various time intervals over two months, during which no degradation product peak has been observed during the analysis of these solutions by the developed method. This was confirmed by analysis of the mass spectra of the solutions. 3.3 clinical application.

Healthy volunteers participated in this study to support the applicability of the developed method to quantify 
TABLE 3: Data of relative recovery of GFX.

\begin{tabular}{|c|c|c|c|c|c|c|}
\hline \multirow{2}{*}{ Sample no. } & \multicolumn{2}{|c|}{ LQC $\left(15 \mathrm{ng} \mathrm{mL}^{-1}\right)$} & \multicolumn{2}{|c|}{ MQC (150 ng mL $\left.{ }^{-1}\right)$} & \multicolumn{2}{|c|}{ HQC (400 ng mL $\left.{ }^{-1}\right)$} \\
\hline & Found & $R^{\mathrm{a}}(\%)$ & Found & $R(\%)$ & Found & $R(\%)$ \\
\hline Mean $^{\mathrm{b}}$ & 14.89 & 99.27 & 150.26 & 100.17 & 402.33 & 100.58 \\
\hline Standard deviation (SD) & 0.31 & 2.07 & 2.74 & 1.83 & 2.85 & 0.71 \\
\hline Precision (CV) (\%) & 2.08 & 2.08 & 1.82 & 1.82 & 0.71 & 0.71 \\
\hline
\end{tabular}

${ }^{a}$ Relative recovery.

${ }^{\mathrm{b}}$ Average of six determinations.

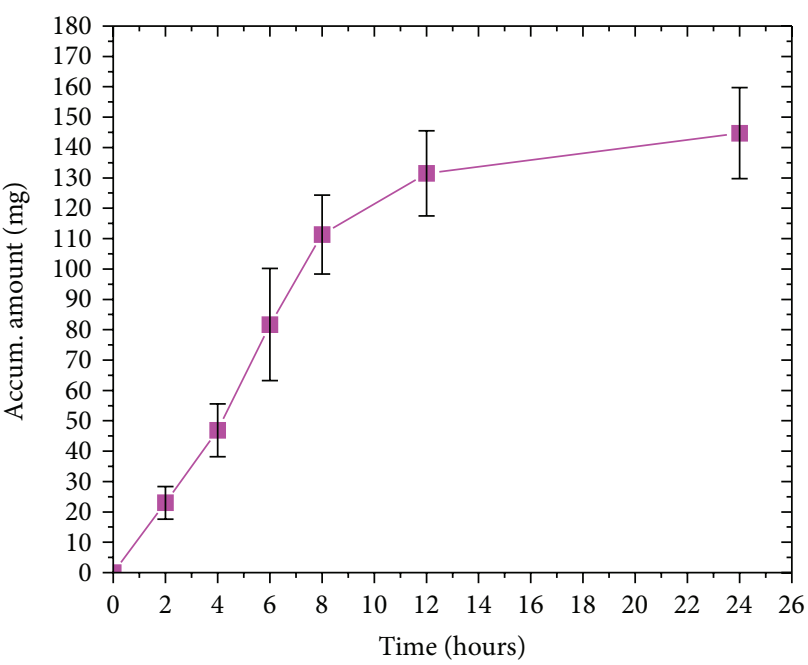

FIGURE 4: The cumulative amount-time curve of GFX in urine from healthy subjects after oral administration of $320 \mathrm{mg}$ GFX tablet $(n=$ $26)$, (mean $\pm S D)$.

GFX for pharmacokinetic purposes in bioequivalence and/or bioavailability studies. Each volunteer was administered a single dose of Factive tablets equivalent to $320 \mathrm{mg}$ gemifloxacin/tablet (Oscient Pharmaceuticals, USA). Urine samples were collected prior to the administration and 2, 4, 6, 8,12 and $24 \mathrm{~h}$ after administration. Figure 4 illustrates the profile of urine cumulative amount-time curve of GFX. The concentration in urine ranged between 15 and $160 \mathrm{ng} \mathrm{mL}^{-1}$ for GFX. The developed method was successfully used to quantify GFX concentration in human urine following GFX oral administration, and it can be successfully applied to the estimation of GFX in any proposed pharmacokinetic study.

\section{Conclusion}

The optimized LC-MS/MS method was validated for the quantification of gemifloxacin in human urine with good linearity in the range of $5-500 \mathrm{ng} \mathrm{mL}^{-1}$. The assay has a simple and rapid procedure for sample preparation and a rapid run time of $4 \mathrm{~min}$. This demonstrated a more ecofriendly elution with reduced consumption of organic solvent along with cost reduction for running the method. The validated method described here, utilizing an isocratic elution and positive ionization tandem MS detection is a rapid, robust, highly selective, and sufficiently sensitive method. Its applicability was demonstrated by analyzing GFX in urine from healthy volunteers. The method can be useful for therapeutic drug monitoring of GFX in urine as well as further pharmacokinetic studies of GFX.

\section{Acknowledgment}

The authors would like to extend their sincere appreciation to the Deanship of Scientific Research at the King Saud University for funding this work through the Research Group Project no. RGP-VPP-322.

\section{References}

[1] G. Y. Lesher, E. J. Froelich, M. D. Gruett, J. H. Bailey, and R. P. Brundage, "1, 8-naphthyridine derivatives. A new class of chemotherapeutic agents," Journal of medicinal and pharmaceutical chemistry, vol. 91, pp. 1063-1065, 1962.

[2] H. Lopez, D. Stepanik, V. Vilches et al., "Comparative in vitro activity of gemifloxacin against gram-positive and gramnegative clinical isolates in Argentina," Diagnostic Microbiology and Infectious Disease, vol. 40, no. 4, pp. 187-192, 2001.

[3] J. Oh, K. Paek, M. Ahn et al., "In vitro and in vivo evaluations of LB20304, a new fluoronaphthyridone," Antimicrobial Agents and Chemotherapy, vol. 40, no. 6, pp. 1564-1568, 1996.

[4] M. G. Cormican and R. N. Jones, "Antimicrobial activity and spectrum of LB20304, a novel fluoronaphthyridone," Antimicrobial Agents and Chemotherapy, vol. 41, no. 1, pp. 204-211, 1997.

[5] A. F. Hohl, R. Frei, V. Pünter et al., "International multicenter investigation of LB20304, a new fluoronaphthyridone," Clinical Microbiology and Infection, vol. 4, no. 5, pp. 280-284, 1998.

[6] T. A. Davies, L. M. Kelly, G. A. Pankuch, K. L. Credito, M. R. Jacobs, and P. C. Appelbaum, "Antipneumococcal activities of gemifloxacin compared to those of nine other agents," Antimicrobial Agents and Chemotherapy, vol. 44, no. 2, pp. 304310, 2000.

[7] T. A. Davies, L. M. Kelly, D. B. Hoellman et al., "Activities and postantibiotic effects of gemifloxacin compared to those of 11 other agents against Haemophilus influenzae and Moraxella catarrhalis," Antimicrobial Agents and Chemotherapy, vol. 44, no. 3, pp. 633-639, 2000.

[8] J. Dubois and C. St-Pierre, "Comparative in vitro activity and post-antibiotic effect of gemifloxacin against Legionella spp," Journal of Antimicrobial Chemotherapy, vol. 45, no. 4, pp. 41-46, 2000.

[9] P. M. Roblin, T. Reznik, A. Kutlin, and M. R. Hammerschlag, "In vitro activities of gemifloxacin (SB 265805, LB20304) against recent clinical isolates of Chlamydia pneumoniae," Antimicrobial Agents and Chemotherapy, vol. 43, no. 11, pp. 2806-2807, 1999. 
[10] P. C. T. Hannan and G. Woodnutt, "In vitro activity of gemifloxacin (SB 265805; LB20304a) against human mycoplasmas," Journal of Antimicrobial Chemotherapy, vol. 45, no. 3, pp. 367$369,2000$.

[11] J. M. Blondeau and G. S. Tillotson, "Gemifloxacin for the treatment of uncomplicated urinary infections (acute cystitis)," Ginecologia y Obstetricia de Mexico, vol. 77, no. 12, pp. 573-582, 2009.

[12] J. M. Blondeau, "Fluoroquinolones: mechanism of action, classification, and development of resistance," Survey of Ophthalmology, vol. 49, supplement 2, pp. S73-S78, 2004.

[13] C. M. Oliphant and G. M. Green, "Quinolones: a comprehensive review," American Family Physician, vol. 65, no. 3, pp. 455-464, 2002.

[14] S. Souverain, S. Rudaz, and J. Veuthey, "Matrix effect in LCESI-MS and LC-APCI-MS with off-line and on-line extraction procedures," Journal of Chromatography A, vol. 1058, no. 1-2, pp. 61-66, 2004.

[15] I. Marchi, S. Rudaz, M. Selman, and J. Veuthey, "Evaluation of the influence of protein precipitation prior to on-line SPE-LCAPI/MS procedures using multivariate data analysis," Journal of Chromatography B, vol. 845, no. 2, pp. 244-252, 2007.

[16] D. C. Charan and S. Satyabrata, "Simple and rapid spectrophotometric estimation of gemifloxacin mesylate in bulk and tablet formulations," International Journal of PharmTech Research, vol. 3, no. 1, pp. 133-135, 2011.

[17] R. R. Das and P. P. Sunita, "Validated UV-spectrophotometric methods for determination of gemifloxacin mesylate in pharmaceutical tablet dosage forms," Journal of Chemistry, vol. 7, pp. S344-S348, 2010.

[18] M. V. Krishna and D. G. Sankar, "Utility of $\delta$ and $\pi$-acceptors for the spectrophotometric determination of gemifloxacin mesylate in pharmaceutical formulations," Journal of Chemistry, vol. 5, no. 3, pp. 493-498, 2008.

[19] M. V. Krishna and D. G. Sankar, "Spectrophotometric determination of gemifloxacin mesylate in pharmaceutical formulations through ion-pair complex formation," Journal of Chemistry, vol. 5, no. 3, pp. 515-520, 2008.

[20] D. Madhuri, K. B. Chandrasekhar, N. Devanna, and G. Somasekhar, "Direct and derivative spectrophotometric estimation of gemifloxacin by chelation with palladium(II) ion," Rasayan Journal of Chemistry, vol. 3, no. 1, pp. 159-165, 2010.

[21] C. S. Paim, F. Führ, M. Steppe, and E. E. S. Schapoval, "Gemifloxacin mesylate: UV spectrophotometric method for quantitative determination using experimental design for robustness," Quimica Nova, vol. 35, no. 1, pp. 193-197, 2012.

[22] S. Sahu, S. K. Patro, U. L. Narayan, and B. Garnaik, "Ion-pair spectrophotometric estimation of gemifloxacin," Pharmaceutical Methods, vol. 3, pp. 26-30, 2012.

[23] S. E. K. Tekkeli and A. Önal, "Spectrofluorimetric methods for the determination of gemifloxacin in tablets and spiked plasma samples," Journal of Fluorescence, vol. 21, no. 3, pp. 1001-1007, 2011.

[24] S. A. M. Ebraheem, A. A. Elbashir, and H. Y. Aboul-Enein, "Spectrophotometric methods for the determination of gemifloxacin in pharmaceutical formulations," Acta Pharmaceutica Sinica B, vol. 1, pp. 248-253, 2011.

[25] Q. Xia, Y. Yang, and M. Liu, "Spectrofluorimetric determination of fluoroquinolones in honey with 2, 3-dichloro-5, 6-dicyano-1, 4-benzoquinone in the presence of $\beta$-cyclodextrin," Journal of Fluorescence, vol. 23, pp. 1-11, 2013.
[26] A. A. Elbashir, B. Saad, A. S. M. Ali, K. M. M. Al-Azzam, and H. Y. Aboul-Enein, "Validated stability indicating assay of gemifloxacin and lomefloxacin in tablet formulations by capillary electrophoresis," Journal of Liquid Chromatography and Related Technologies, vol. 31, no. 10, pp. 1465-1477, 2008.

[27] R. N. Rao, C. G. Naidu, K. G. Prasad, R. Padiya, and S. B. Agwane, "Determination of gemifloxacin on dried blood spots by hydrophilic interaction liquid chromatography with fluorescence detector: application to pharmacokinetics in rats," Biomedical Chromatography, vol. 26, pp. 1534-1542, 2012.

[28] C. Vinodhini, K. Chitras, A. S. S. Annie, I. Marbaniang, A. K. Singh, D. Ashok et al., "Determination of gemifloxacin in tablets by reverse phase high performance liquid chromatography," Indian Drugs, vol. 46, pp. 71-73, 2009.

[29] B. M. H. Al-Hadiya, A. A. Khady, and G. A. E. Mostafa, "Validated liquid chromatographic-fluorescence method for the quantitation of gemifloxacin in human plasma," Talanta, vol. 83, pp. 110-116, 2010.

[30] W. Lee and C. Y. Hong, "Direct liquid chromatographic enantiomer separation of new fluoroquinolones including gemifloxacin," Journal of Chromatography A, vol. 879, no. 2, pp. 113$118,2000$.

[31] A. Allen, E. Bygate, M. Vousden et al., "Multiple-dose pharmacokinetics and tolerability of gemifloxacin administered orally to healthy volunteers," Antimicrobial Agents and Chemotherapy, vol. 45, no. 2, pp. 540-545, 2001.

[32] C. S. Paim, F. Fuhr, S. D. Miron et al., "Stability-indicating LC assay and determination of system suitability limits with a robustness test of gemifloxacin mesylate in tablets," Current Analytical Chemistry, vol. 8, pp. 269-276, 2010.

[33] A. R. Rote and S. P. Pingle, "Reverse phase-HPLC and HPTLC methods for determination of gemifloxacin mesylate in human plasma," Journal of Chromatography B, vol. 877, no. 29, pp. 37193723, 2009.

[34] N. Sultana, M. S. Arayne, S. Shamim, M. Akhtar, and S. Gul, "Validated method for the determination of gemifloxacin in bulk, pharmaceutical formulations and human serum by RPHPLC: in vitro applications," Journal of the Brazilian Chemical Society, vol. 22, no. 5, pp. 987-992, 2011.

[35] B. V. d. Araújo, J. V. Laureano, L. D. Grünspan, T. D. Costa, and L. Tasso, "Validation of an efficient LC-microdialysis method for gemifloxacin quantitation in lung, kidney and liver of rats," Journal of Chromatography B, vol. 919, pp. 62-66, 2013.

[36] M. Gumustas and S. A. Ozkan, "Simple, sensitive and reliable LC-DAD method of gemifloxacin determination in pharmaceutical dosage forms," Turkish Journal of Pharmaceutical Sciences, vol. 9, pp. 161-170, 2012.

[37] U. S. Chakrabarty, A. Das, U. Bhaumik et al., "Rapid and sensitive LC method for the analysis of gemifloxacin in human plasma," Chromatographia, vol. 69, no. 9-10, pp. 853-858, 2009.

[38] B. Roy, A. Das, U. Bhaumik et al., "Determination of gemifloxacin in different tissues of rat after oral dosing of gemifloxacin mesylate by LC-MS/MS and its application in drug tissue distribution study," Journal of Pharmaceutical and Biomedical Analysis, vol. 52, no. 2, pp. 216-226, 2010.

[39] E. Doyle, S. E. Fowles, D. F. McDonnell, and S. A. White, "Rapid determination of gemifloxacin in human plasma by high-performance liquid chromatography-tandem mass spectrometry," Journal of Chromatography B, vol. 746, no. 2, pp. 191198, 2000.

[40] V. R. Robledo and W. F. Smyth, "A study of the analytical behaviour of selected new molecular entities using electrospray 
ionisation ion trap mass spectrometry, liquid chromatography, gas chromatography and polarography and their determination in serum at therapeutic concentrations," Analytica Chimica Acta, vol. 623, no. 2, pp. 221-230, 2008.

[41] H. Lee, T. E. Peart, and M. L. Svoboda, "Determination of ofloxacin, norfloxacin, and ciprofloxacin in sewage by selective solid-phase extraction, liquid chromatography with fluorescence detection, and liquid chromatography-tandem mass spectrometry," Journal of Chromatography A, vol. 1139, no. 1, pp. 45-52, 2007.

[42] S. I. Cho, J. Shim, M. Kim, Y. Kim, and D. S. Chung, "Online sample cleanup and chiral separation of gemifloxacin in a urinary solution using chiral crown ether as a chiral selector in microchip electrophoresis," Journal of Chromatography A, vol. 1055, no. 1-2, pp. 241-245, 2004.

[43] F. Zhao, W. Zhao, and W. Xiong, "Chemiluminescence determination of gemifloxacin based on diperiodatoargentate (III)sulphuric acid reaction in a micellar medium," Luminescence, vol. 28, pp. 108-113, 2012.

[44] A. M. Al-Mohaimeed, S. A. Al-Tamimi, N. A. Alarfaj, and F. A. Aly, "New coated wire sensors for potentiometric determination of gemifloxacin in pure form, pharmaceutical formulations and biological fluids," International Journal of Electrochemical Science, vol. 7, pp. 12518-12530, 2012.

[45] ICH Guidance for Industry, QR1 Validation of Analytical Procedures: Methodology, ICH, Geneva, Switzerland, 1996.

[46] C. Jiménez, R. Ventura, and J. Segura, "Validation of qualitative chromatographic methods: strategy in antidoping control laboratories," Journal of Chromatography B, vol. 767, no. 2, pp. 341-351, 2002. 

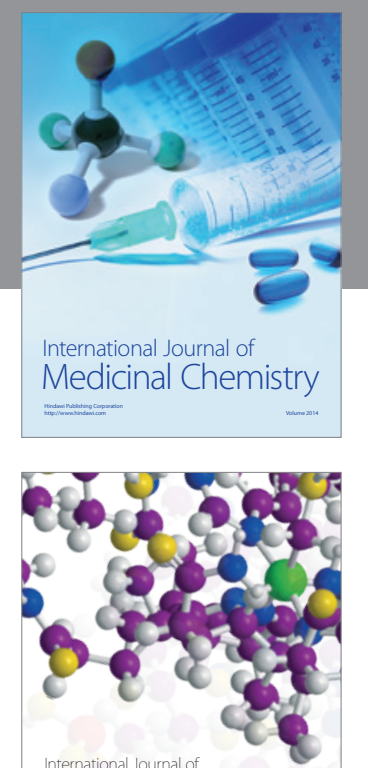

\section{Carbohydrate} Chemistry

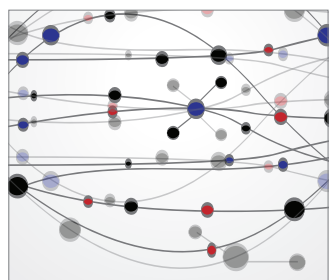

The Scientific World Journal
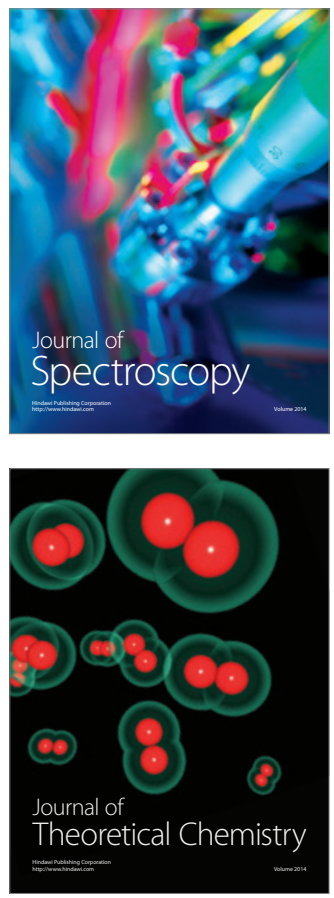
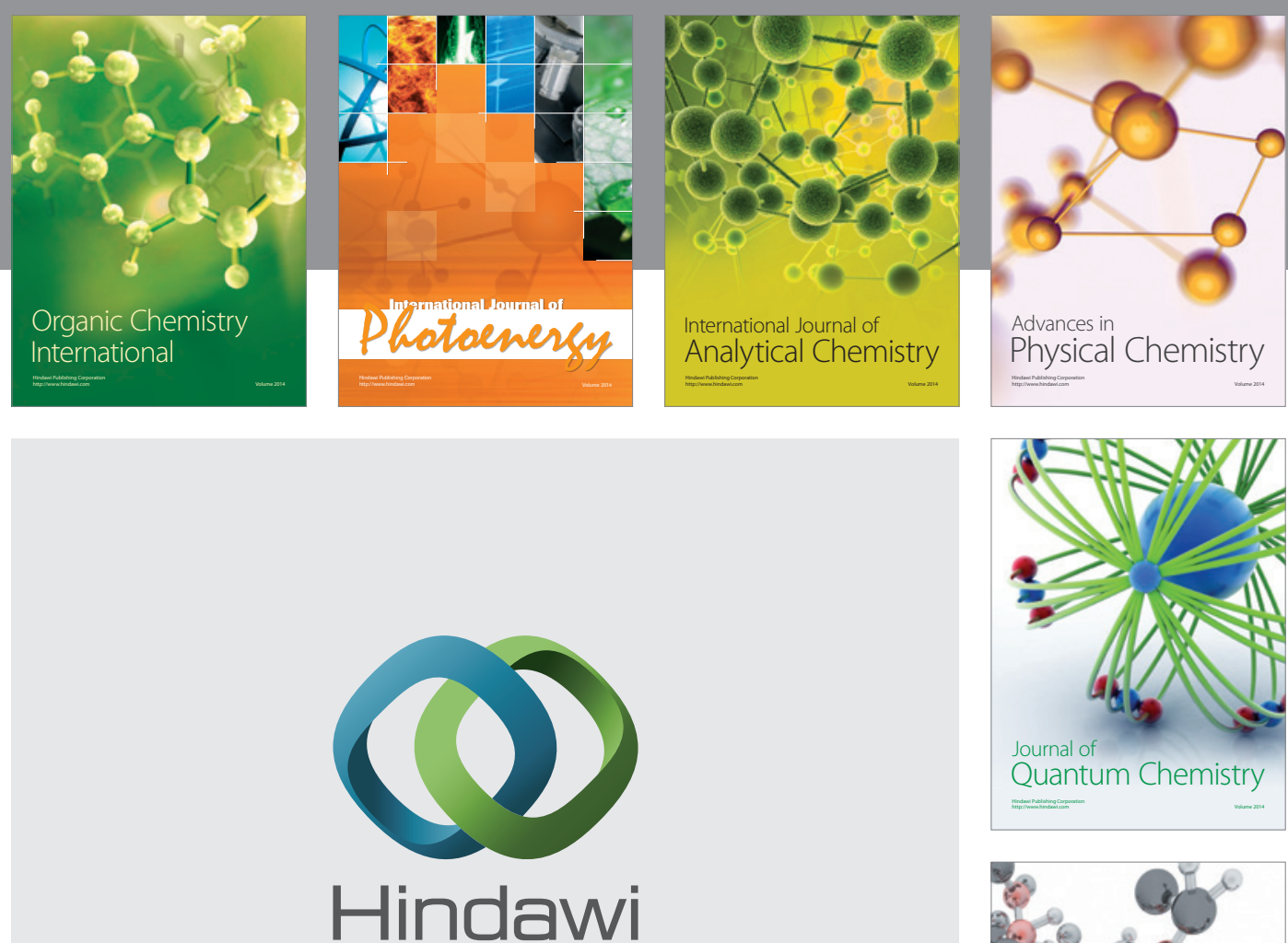

Submit your manuscripts at

http://www.hindawi.com

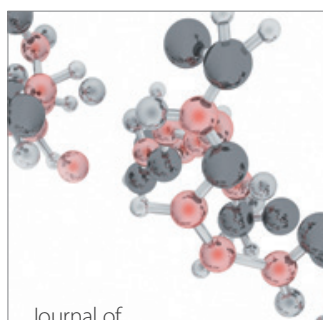

Analytical Methods

in Chemistry

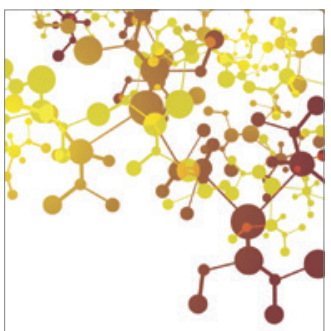

Journal of

Applied Chemistry

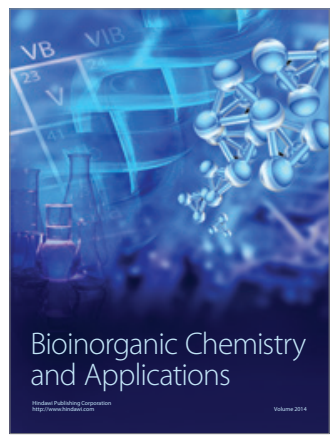

Inorganic Chemistry
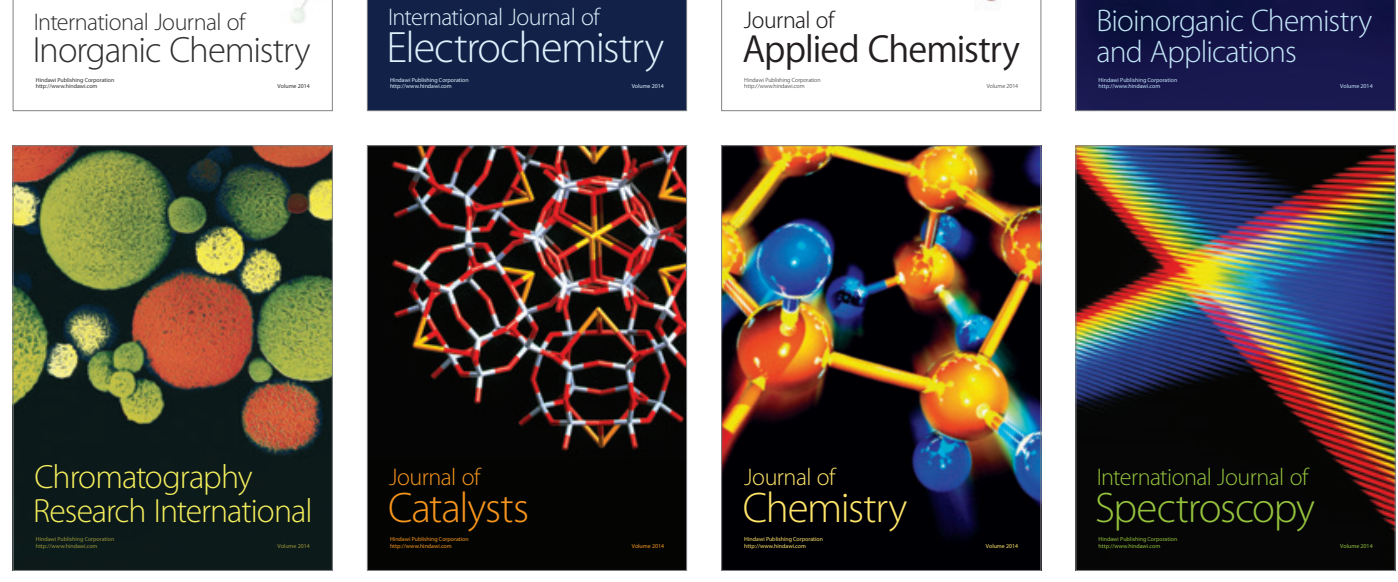